\title{
Prolonged and exclusive breastfeeding of infants did not reduce growth by 12 months of age
}

\author{
Kramer MS, Guo T, Platt RW, et al for the PROBIT Study Group. Breastfeeding and infant growth: biology or bias? \\ Pediatrics 2002;110:343-7.
}

\section{QUESTION: Is a breastfeeding promotion intervention shown to increase duration and exclusivity of breast feeding associated with lower infant weight and length?}

\section{Design}

Cluster randomised (cluster allocation concealed), unblinded, controlled trial with 12 months of follow up.

Sources of funding: Thrasher Research Fund; the National Health Research and Development Program (Health Canada) UNICEF.

For correspondence: Dr M S Kramer, McGill University Faculty of Medicine Montreal, Quebec,

Canada.

michael.kramer@mcgill.ca

\section{Setting}

32 maternity hospitals and their affiliated clinics in Belarus.

\section{Participants}

Mother-infant pairs were enrolled if the mother intended to breast feed, had no illnesses contraindicating breast feeding, and had given birth to a healthy, singleton infant who had a gestational age $\geq 37$ weeks, birth weight $\geq 2500 \mathrm{~g}$, and a 5 minute Apgar score $\geq 5$. 17046 mother-infant pairs were enrolled, and 16471 $(97 \%)$ completed follow up. 31 sites were included in the analysis ( 1 site was omitted because data were falsified).

Effect of Baby Friendly Health Initiative breastfeeding intervention v standard care (control) on infant weight and body length

\begin{tabular}{|c|c|c|c|c|c|}
\hline Outcomes & $\begin{array}{l}\text { Follow up } \\
\text { (months) }\end{array}$ & Intervention & Control & Difference & p value \\
\hline \multirow[t]{6}{*}{ Mean body weight (g) } & 1 & 4341 & 4280 & 61 & 0.001 \\
\hline & 2 & 5258 & 5170 & 88 & $<0.001$ \\
\hline & 3 & 6153 & 6047 & 106 & $<0.001$ \\
\hline & 6 & 8131 & 8042 & 89 & $<0.001$ \\
\hline & 9 & 9509 & 9451 & 58 & 0.002 \\
\hline & 12 & 10564 & 10571 & -7 & 0.726 \\
\hline \multirow[t]{6}{*}{ Mean body length $(\mathrm{cm})$} & 1 & 54.79 & 54.63 & 0.16 & 0.258 \\
\hline & 2 & 57.89 & 57.75 & 0.32 & 0.030 \\
\hline & 3 & 61.13 & 60.63 & 0.50 & 0.001 \\
\hline & 6 & 67.36 & 66.90 & 0.46 & 0.002 \\
\hline & 9 & 71.93 & 71.62 & 0.31 & 0.039 \\
\hline & 12 & 75.93 & 75.75 & 0.18 & 0.226 \\
\hline
\end{tabular}

\section{Intervention}

Study sites were allocated to an intervention based on the Baby Friendly Hospital Initiative (BFHI) $(n=16)$ or to standard care (control) $(n=15)$. The intervention involved training midwives, nurses, and physicians in the BFHI lactation management course, which emphasised methods to maintain lactation, promote exclusive and prolonged breast feeding, and solve common problems.

\section{Main outcome measures}

Weight, body length, and head circumference measured at 1, 2, 3, 6, 9, and 12 months of follow up.

\section{Main results}

Analysis was by intention to treat. Infants in the intervention group were more likely to be breast fed than infants in the control group during their first 12 months and were more likely to be exclusively breast fed at 3 months $(43 \% v 6.4 \% \mathrm{p}<0.001)$. Mean body weight was greater in the intervention group than in the control group at 1 month; the difference increased through 3 months, declined somewhat thereafter, and disappeared by 12 months (table). Body length followed a similar overall pattern as weight (table). The groups did not differ for head circumference at any time during follow up.

\section{Conclusion}

A breastfeeding promotion intervention shown to increase duration and exclusivity of breast feeding was not associated with deficits in weight or length during the first year of life.

\section{COMMENTARY}

In this study by Kramer $e$ al, the long held view that prolonged and exclusive breast feeding is a causal factor in reduced infant growth is tested. This is the first study to look at this association using a randomised controlled trial design. Previous studies had used observational designs, which had problems such as selection bias, reverse causality, and confounding effects.

The investigators took the opportunity to examine infant growth after exposure to a breastfeeding promotion intervention. The authors caution that infant growth measurements were assessed independently by each of the 32 clusters of study participants and were not standardised. This approach introduces a potential source of random error, particularly when assessing parameters such as infant length where measurements vary, even when using the same technique.

Even with a cluster randomisation strategy, some interaction (contamination) between study sites could exist. Chiefs of Obstetrics at sites not randomised to the intervention likely had some awareness about the WHO/UNICEF BFHI, but the increase in breastfeeding practice at the intervention sites clearly shows the effectiveness of the study intervention.

Although one of the 32 study sites was excluded from the analyses because data were falsified, a mechanism was in place to correlate the study data sheets with maternal records and interviews at the study sites. Overall, strong agreement between the study data sheets and hospital records was shown in the remaining sites.

The importance of randomised trials in uncovering sources of bias associated with observational studies is clear. When data were analysed by reconstructing the groups according to breastfeeding practices and ignoring randomisation, the findings supported previous observational studies, suggesting that exclusive breast feeding slowed growth.

Although the findings relate to the Belarus population, they are also relevant for maternal child nurses in higher income countries of the West. Strong evidence is provided that exclusive and prolonged breast feeding in healthy humans will enhance infant growth during the first 3 months of life and that growth patterns during the first year will not be affected. This evidence should instil confidence into practitioners and parents alike that exclusive breast feeding is sufficient for optimal infant growth. 